\title{
C. Zur erklärung und kritik der schriftsteller.
}

\section{Zu Platon's Phaedon.}

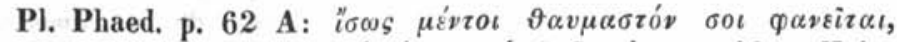

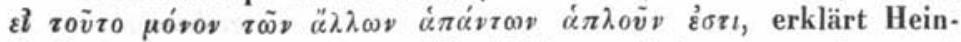
dorf: forlasse tamen mirum tibi videbilur, si hoc unum de celeris omnibus simpliciter verum sit et sine ulla exceptione (sc. mori melius esse, quam vivere), und Stallbaum stimmt ihm bei. Aber ein logischer zusammenhang kommt nur dann heraus, wenn gerade umgekehrt gedeutet wird: vivere melius esse, quam esse mortuum. Es soll nämlich dargethan werden, dass selbstmord unstatthaft sei. Diese unstatthaftigkeit würde leicht erhellen, wenn man voraussetzen dürfte, der tod sei jedesmal ein übel und das leben jedesmal ein gut. Aber, sagt Sokrates, diese voraussetzung würde dir mit recht als seltsam und verwunderlich erscheinen, denn wie sollte es nicht auch hier, selbst wenn der vorzug des lebens vor dem tode als regel gelten kann, ausnahmsfälle geben, in denen der tod besser ist, als das leben. Giebt es aber solche, dann ist auffallend, dass dennoch die selbsttödtung als der nächste weg zur erlangung dieses gutes unerlaubt sein soll. So besteht ein strenger gedankenfortschritt; bei der andern deutung geräth alles in unlösbare verwirrung.

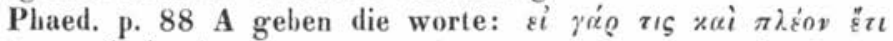

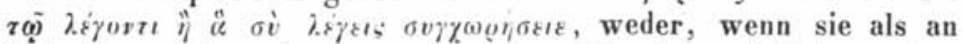
Sokrates, noch auch, wenn sie als an Simmias gerichtet verstanden werden, einen vernünftigen sinn. Dass Simmias früher den satz von der präexistenz der seele gebilligt hatte, kann hier nicht in betracht kommen; denn das hat Simmias nicht in diesem gedankengang zugestanden, in welchem er vielmehr die seele als harmonie an den leib bindet; an das frühere zugeständniss zu erinnern, ist dem Sokrates (p. 92 A ff.) vorbehalten. Die conjectur Schleiermachers, der das $i \hat{i}$ auswirft, erscheint als durchaus nothwendig. Die worte sind an Sokrates gerichtet. Sehr schwach ist Stallbaums einwurf, dann luätte $\sigma o i$ genïgt. Denn viel passender, als das einfache $\sigma o i$, war der verallgemeinernde ausdruck:

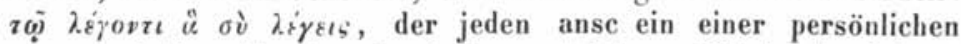
gegnerschaft entfernt und rein das sachliche verhältniss bezeichnet.

Phaed. p. 92 D vertheidigen Heindorf, Stalibaum und andere

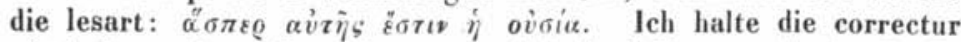
$\alpha \dot{v} i \dot{y}$ für nothwendig. Denn es wird die frühere stelle (p. 76 D, E und 77 A) citirt, wo nach der recapitulation des Simmias die gewissheit von der präexistenz der seele mit der gewissheit von der existenz der ideen gleichgestellt wird. In der vorangehenden, durch Sokrates selbst gegebenen recapitulation wird zwar auch gesagt, dass der schluss, der die präexistenz der seele ergiebt, auf der existenz der ideen und auf der beziehung der wahrneh- 
mungen auf sie als vorher gewusste beruhe; aber dann wird auch dort die gewissheit von der präexistenz der seele mit der gewissheit von der existenz der ideen gleichgestellt. Also musste auch p. $92 \mathrm{D}$, wo nur der frühere gedanke wieder aufgenommen werden soll, das gleiche geschehen. Dazu kommt, dass der mehr-

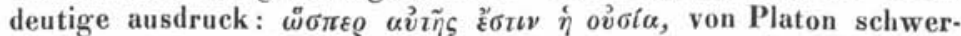
lich gewählt worden wäre, um das gewusstwerden der ideen durch die seele zu bezeichnen; dieser ausdruck könnte nicht durch das $\grave{\eta} \mu \varepsilon \tau \varepsilon \hat{\varepsilon} \alpha \nu$ ov $\sigma \alpha \nu$ an der früheren stelle gerechtfertigt werden,

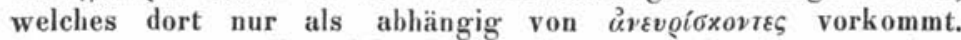
Strenggenommen ist nicht die ideelle wesenheit selbst, sondern nur das wissen von ihr im besitz der seele, und nur vermöge einer durch den zusammenhang gerechtfertigten metapher kann sie selbst als besitzthum der seele bezeichnet werden.

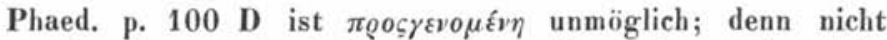
die parusie oder gemeinschaft, d. h. das hinzugetretensein, tritt

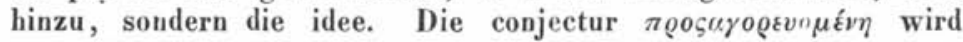
zwar durch analogien gestützt, die freilich vielmehr auf noosuyo-

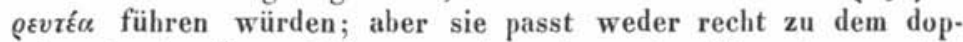
pelausdruck: $: \pi \eta \eta$ x $\iota \grave{i} \delta \pi \omega \varsigma$, noch auch zu den folgenden worten, dass Sokrates darüber nichts versichern wolle, was beides vielmehr auf die art des hinzutretens, als auf die art der benennung

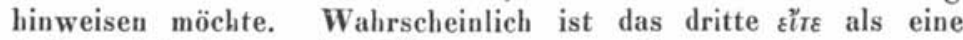
irrige wiederholung der früheren auszuwerfen und dann $\pi \varrho \circ \varsigma \gamma \varepsilon$. vopḱvov zu lesen: auf welchem wege gleichsam und in welcher weise jenes an sich schöne hinzugetreten sein mag.

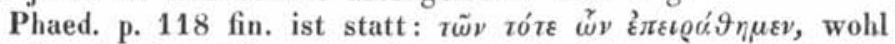

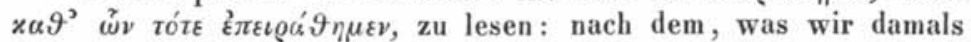

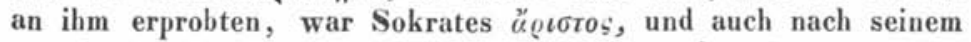

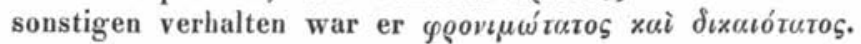

Königsberg in Pr.

Fr, Ueberioeg.

\section{Emendationes ad grammaticos Graecos.}

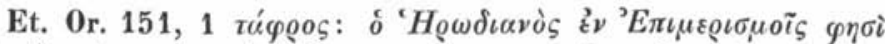

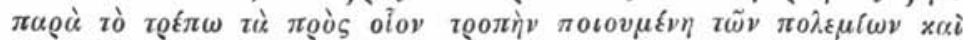

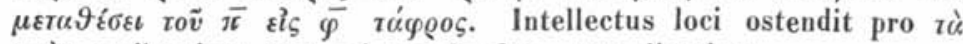

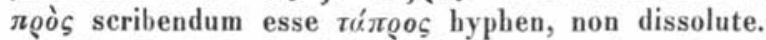

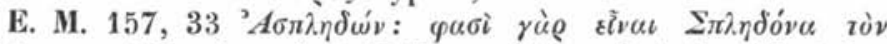

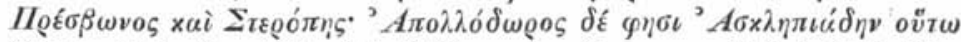

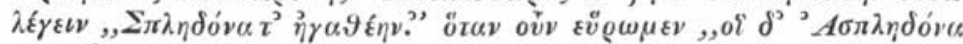

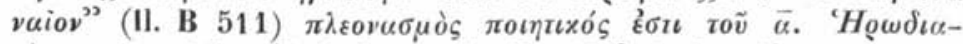
vò $\pi \varepsilon \rho i{ }^{2} \pi u \vartheta \tilde{\omega} v$. Lehrsius in Anall. $429^{\prime} A \sigma \times \lambda \eta \pi u \dot{\delta} \delta \eta v$ corruptum

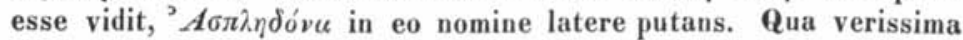

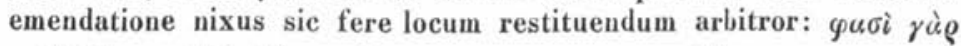

$$
\text { rhilologus. Xx. Jahrg. } 3 .
$$

